\title{
Estudo das técnicas de preenchimento para dados de fluxo de metano em uma área de arroz irrigado por inundação
}

\author{
Study of filling techniques for methane flow data in an area of flooded rice
}

immediate

${ }^{1}$ Lucas Augusto Fagundes, ${ }^{1}$ Cristiano Maboni, ${ }^{1}$ Maria Eduarda Pinheiro, ${ }^{1}$ Josue Sehnem, ${ }^{1}$ Marcelo Diaz e ${ }^{1}$ Debora Regina Roberti

\author{
${ }^{1}$ Universidade Federal de Santa Maria
}

\section{Resumo}

Estudos sobre a emissão de gás metano $\left(\mathrm{CH}_{4}\right)$ em ecossistemas agrícolas têm recebido atenção da comunidade científica pois tem um importante papel no efeito estufa. Áreas de agricultura de irrigação por inundação, como é o caso do arroz. irrigado, representam uma fonte considerável de emissões de metano (de 12 a 26\% de toda a emissão antropogênica de $\mathrm{CH}_{4}$ do mundo). Estimativas das emissões de $\mathrm{CH}_{4}$ têm sido realizadas também através da técnica covariância dos vórtices. Embora esta metodologia faça medidas contínuas das trocas de metano entre o ecossistema e a atmosfera, falha nas coletas dos dados podem ocorrer, pois o sistema é bastante sensível, principalmente às intempéries. O preenchimento das falhas na estimativa de metano é imprescindível para obtermos uma quantificação diária ou sazonal das emissões. Neste estudo, diferentes técnicas de preenchimento de falhas foram testadas nos fluxos de $\mathrm{CH}_{4}$, obtidos por meio da técnica de covariância dos vórtices sobre uma cultura de arroz irrigado. Os dados experimentais foram obtidos em Cachoeira do Sul - RS no período de 20/11/2015 a 30/04/2016. A técnica de preenchimento Look-Up Table (LUT), que consiste em preencher falhas utilizando médias de valores de fluxo para os períodos que possuam condições atmosféricas similares, foi, na média, a técnica que melhor preencheu os dados de fluxos de metano.

Palavras-chave: Fluxo de metano, Preenchimento de falhas, MDV, LUT, LI.

\begin{abstract}
Studies on the emission of methane gas $\left(\mathrm{CH}_{4}\right)$ in agricultural ecosystems have received attention from the scientific community because it plays an important role in the greenhouse effect. Flood irrigation agriculture, such as irrigated rice, represents a considerable source of methane emissions (from 12 to 26 percent of all anthropogenic $\mathrm{CH}_{4}$ emissions in the world). Estimates of $\mathrm{CH}_{4}$ emissions have also been performed through the eddy covariance technique. Although this methodology makes continuous measurements of methane exchanges between the ecosystem and the atmosphere, failure to collect data can occur because the system is quite sensitive, especially in the hard weather conditions. The gap filling in the methane data are essential to obtain a daily or seasonal quantification of the emissions. In this study, different gap filling techniques are used to fill the $\mathrm{CH}_{4} \mathrm{fluxes}_{\mathrm{s}}$ obtained by eddy covariance technique in an irrigated rice crop. The experimental data were obtained in Cachoeira do Sul - RS in the period from 11/20/2015 to 04/30/2016. The Look-Up Table (LUT), which consists of filling gaps using averages of flux values for periods with similar atmospheric conditions, was the technique that best close the gap in methane fluxes data.
\end{abstract}

Keywords: Methane Flux, Gap Filling, MDV, LUT, LI. 


\section{Introdução}

A concentração atmosférica de $\mathrm{CH}_{4}$ tem apresentado os maiores níveis desde os últimos 800.000 anos (IPCC (2013)), como esgotos, trato gastro intestinal de alguns animais e sedimentos aquáticos (Alvalá et al. (1999)), como áreas de cultivo de arroz irrigado. A produção de arroz irrigado por inundação é responsável por 12-26\% de toda a emissão antropogênica de $\mathrm{CH}_{4}$ do mundo (Alberto et al. (2014)). O Brasil é um dos principais produtores de arroz do mundo (CONAB: Companhia Nacional de Abastecimento (2016)), sendo cultivado principalmente de forma irrigado por inundação.

Estimativas das emissões de metano por ecossistemas pode ser obtido pelo método de eddy-covariance, que se tornou popular nas últimas décadas e atualmente é a mais indicada para as trocas de energia e gases entre a superfície e a atmosfera, em particular vapor de água (H2 O), $\mathrm{CO}_{2}$ e $\mathrm{CH}_{4}$ (Falge et al. (2001)).

O método de Eddy-Covariance (EC) têm sido utilizado para estudos dos fluxos de metano por vários autores (Bhattacharyya et al. (2014); Detto et al. (2011); Alberto et al. (2014). Embora esta metodologia faça medidas contínuas das trocas de metano entre o ecossistema e a atmosfera, falha na coleta dos dados podem ocorrer. A instrumentação empregada na EC é muito sensível a interferências da precipitação, fauna (pouso de pássaros), manutenções e falta de energia, acarretando em falhas na série temporal dos fluxos (BURBA G. e ANDERSON D. (2005)). Para obtermos uma série contínua de fluxo, é necessária a aplicação de técnicas de preenchimento de dados (Dengel et al. (2013); Falge et al. (2001). O princípio de funcionamento da maioria das técnicas de preenchimento de falhas de fluxo está diretamente relacionado ao modo como o ecossistema se comporta em relação as variáveis do meio ambiente, tais como temperatura, radiação solar, umidade relativa, etc. (Taiz e Zeiger (2006)).

Este trabalho tem como objetivo analisar diferentes técnicas de preenchimento de falhas nos fluxos de $\mathrm{CH}_{4}$, obtidos por meio da EC. Foram analisadas três combinações de técnicas: Interpolação Linear (LI), Mean Diurnal Variation (MDV), Look-Up Table (LUT).

\section{Materiais e Métodos}

\subsection{Dados Experimentais}

Os dados experimentais de metano foram obtidos de uma torre micrometeorológica localizada sobre uma cultura de arroz irrigado em Cachoeira do Sul no estado do Rio Grande do Sul (latitude: -30,2771; Longitude: -53,1479; Altitude: 40,5 metros) no período de 20/11/2015 a 30/04/2016.

Na safra 2015-2016, o plantio de arroz neste sítio ocorreu com a cultivar 118 comum EPAGRI no dia 14 de novembro de 2015 e a colheita ocorreu dia 16 de abril de 2016. A produtividade média da área foi de $0,4 \mathrm{Kg} \mathrm{m}-2$. A inundação do solo ocorre antes do plantio e foi mantida com aproximadamente $10 \mathrm{~cm}$ de lâmina d'água de forma constante até a colheita. O solo é classificado como Planossolo Hidromórfico (Embrapa (2006)). As seguintes medidas de interesse neste trabalho, foram realizadas na área experimental (altura do sensor acima do solo, fabricante, modelo): Gás metano (3m, LiCor, LI-7700), componentes do vento e temperatura do ar (3m, Gill - LICOR); $\mathrm{H}_{2} \mathrm{O} / \mathrm{CO}_{2}$ analisador de gás e pressão (3m, LiCor, LI7500); radiação de onda curta (8 m, Kipp \& Zonen, CMB6); radiação de onda longa (8m; Kipp \& Zonen, CG).

\subsection{Processamentos dos fluxos de metano}

O processamento dos fluxos de metano $\left(\mathrm{fCH}_{4}\right)$ ocorreu utilizando o software EddyPro versão 6.0, LiCor (Lincoln, Nebraska, EUA) com médias de 30 minutos. O mesmo processamento utilizado neste artigo foi o mesmo descrito por (Diaz e Roberti, 2015). Após o processamento os valores de $\mathrm{fCH}_{4}$ menores que $0 \mu$ mol m - $2 \mathrm{~s}-1$ e superiores a $2.5 \mu$ mol m $-2 \mathrm{~s}-1$ foram retirados por não representarem fisicamente o fenômeno das emissões. Para uma classificação dos resultados do coeficiente de determinação $\left(\mathrm{r}^{2}\right)$, foi utilizada a metodologia proposta por (Moffat et al., 2007) que qualifica os valores como: "ruim" $\left(0.5 \leq \mathrm{r}^{2}\right)$, "Médio" $\left(0.5<\mathrm{r}^{2}\right.$ $\leq 0.75)$, "Bom $\left(0.75<\mathrm{r}^{2} \leq 0.85\right)$ e "Muito Bom" $\left(\mathrm{r}^{2}>0.85\right)$

Valores menores que $0 \mu \mathrm{mol} \mathrm{m}{ }^{-2} \mathrm{~s}^{-1}$ e superiores a $2.5 \mu \mathrm{mol} \mathrm{m}^{-2} \mathrm{~s}^{-1}$ foram retirados por não representarem fisicamente o fenômeno das emissões.

\subsection{Métodos para o preenchimento dos fluxos de metano}

A seguir, descrevem-se as técnicas usadas neste estudo:

- A técnica de interpolação linear simples (LI) consiste em interpolar os pontos faltantes com os valores de dados adjacentes a falha. Esse método é indicado para aplicações em falhas pequenas, não superiores há 4 horas (Foken et al. (2004)). Esse método tem como vantagem a não dependência de forçantes meteorológicos para o preenchimento do fluxo de metano. $\mathrm{O}$ preenchimento de falhas utilizando LI foi feito para falhas com o tamanho de $0.5 \mathrm{~h}$ e $4 \mathrm{~h}$. 
Tabela 1: Visão geral sobre a cobertura e disponibilidade de dados no estudo atual. Todos os valores são fluxos de 30 min. Todos os números, exceto os valores percentuais, são dados em número de linhas de dados. Os dados diurnos e noturnos cobrem os períodos entre radiação global maior que zero e onde houve radiação global igual a zero, respectivamente.

\begin{tabular}{|c|c|c|c|c|c|c|c|c|c|}
\hline Sítio & $\begin{array}{c}\text { Dias } \\
(\mathrm{n})\end{array}$ & $\begin{array}{c}100 \% \text { de } \\
\text { cobertura }\end{array}$ & $\begin{array}{c}\text { Cobertura } \\
\text { atual }\end{array}$ & $\begin{array}{c}\text { Cobertura } \\
(\%)\end{array}$ & $\begin{array}{c}\text { Cobertura } \\
(\text { dias })\end{array}$ & $\begin{array}{c}\text { Diurno } \\
(\mathrm{n})\end{array}$ & $\begin{array}{c}\text { Diurno } \\
(\%)\end{array}$ & $\begin{array}{c}\text { Noturno } \\
(\mathrm{n})\end{array}$ & $\begin{array}{c}\text { Noturno } \\
(\%)\end{array}$ \\
\hline $\mathrm{CS}$ & 163 & 7824 & 3671 & 47 & 76 & 2608 & 53 & 1063 & 36 \\
\hline
\end{tabular}

- A técnica Mean Diurnal Variation (MDV) baseia-se na ideia da autocorrelação dos fluxos ao longo do dia (Falge et al. (2001)), ou seja, para mesmos horários em dias próximos os fluxos tendem a ser bem relacionados (condições similares de temperatura do ar $\left(\mathrm{T}_{\text {air }}\right)$. Assim a falha é preenchida com a média para o mesmo horário dos dias adjacentes. O tamanho da janela é fundamental, sabendo que as janelas pequenas podem ter uma média pouco representativa e as janelas longas podem acrescentar muita incerteza devido ao desenvolvimento da cultura. A grande vantagem do método MDV está na não dependência das forçantes atmosféricas. Este método tem um baixo desempenho para dias com condições meteorológicas diferentes (Diaz e Roberti (2015)). No processo de preenchimento com o MDV, foi utilizado uma janela fixa de 7 dias.

- A técnica Look-Up Table (LUT), preenche falhas utilizando médias de valores de fluxo de metano para os períodos que possuam condições atmosféricas similares. Para isso, o método realiza o preenchimento utilizando a média dos fluxos de metano em faixas próximas ao dado faltante que contenham condições similares de radiação global e temperatura. Pode-se agregar mais forçantes, nesta análise. Entretanto, aumentando o número de variáveis aumenta também a dificuldade em encontrar valores de fluxo de metano que tenham todas estas condições similares, podendo assim aumentar a incerteza da técnica (Aubinet et al. (2012)). Na técnica de preenchimento utilizando o LUT, foi utilizado a temperatura variando de $\pm 2.5^{\circ} \mathrm{C}$ com uma janela fixa de \pm 14 dias. Para o correto funcionamento da técnica LUT, não deve haver falhas nas forçantes atmosféricas. Foi preenchido $49 \%$ dos dados atmosféricos do período analisado, o que ocorreu utilizando dados meteorológicas obtidos da estação meteorológica de Santa Maria (INMET) localizada (83 km de Cachoeira do Sul - RS em linha reta).

\subsection{Métodos de análise das técnicas de preenchimento de dados}

A maneira escolhida para avaliar o desempenho dos métodos descritos na seção acima que foi comparando os dados observados $\left(\mathrm{Obs}_{\mathrm{i}}\right)$ com os dados obtidos a partir das técnicas $\left(\mathrm{Tec}_{\mathrm{i}}\right)$ aqui testadas. Para comparação das técnicas, foram criadas lacunas artificiais nos dados $\mathrm{fCH}_{4}$ para poder comparar com os valores previstos pelas técnicas. Foram criadas falhas proporcionais a aproximadamente $10 \%$ a quantidade de dados existentes (Moffat et al. (2007)), a fim de quantificar como os métodos preenchimentos diferentes tipos de tamanho de falhas, como definimos na tabela 2. Deste modo, foram criadas falhas de meia hora, 4 horas e 12 horas para testar os limites das técnicas de preenchimento. Tal distribuição de falhas foi feita separadamente para cada tamanho de lacuna, sendo distribuídas de forma aleatória na série temporal e comparadas. Esse processo foi realizado 100 vezes, de forma distinta o período noturno e diurno, como nos estudos de (Moffat et al., 2007) que foram realizados 100 vezes para o $\mathrm{CO}_{2}$.

Falhas de 30 minutos e 4 horas foram dispostas a não coincidir com falhas já existentes. Para o fim de comparação foram utilizados índices estatísticos, iniciando pelo coeficiente de determinação $\left(\mathrm{r}^{2}\right)$ que determina a variância da variável dependente

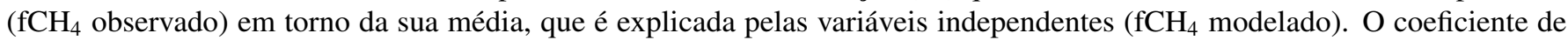
determinação varia entre 0 e 1 , em que quanto mais representativo for o modelo de regressão mais próximo a 1 chegará o valor: pode-se assumir que quanto maior o valor do $\mathrm{r}^{2}$, melhor será a explicação do observado pelo previsto.

$$
r^{2}=\frac{\sum\left(O b s_{i}-\overline{O b} s\right)\left(T e c_{i}-\overline{T e c}\right)^{2}}{\sum\left(O b s_{i}-O \bar{b} s\right)^{2}\left(T e c_{i}-\overline{T e c}\right)^{2}}
$$

Para determinar o erro entre o observado e o previsto foi utilizado a raiz do erro médio quadrático (do inglês Root Mean Square error - RMSE)

$$
R M S E=\sqrt{\frac{1}{n} \sum_{i=1}^{N}\left(T e c_{i}-O b s_{i}\right)^{2}}
$$

No $\mathrm{fCH}_{4}$ foi utilizada a geração de um cenário misto de falhas artificiais tabela 2, distribuídas proporcionalmente em relação a porcentagens de falhas noturnas e diurnas existentes para o sítio. 
Tabela 2: Relação das distribuições das falhas em relação ao seu tamanho para cálculo de esta- tísticas dos modelos no que diz respeito ao período.

\begin{tabular}{|c|c|c|c|}
\hline Tamanho das falhas & 30 minutos & 4 horas & 12 horas \\
\hline Número de ocorrências & 67 & 11 & 6 \\
\hline
\end{tabular}

Figura 1: Fluxo de metano original (Linha preta), gaps criados aleatoriamente no fluxo de metano (linha azul) e fluxo de metano estimado pela técnica de LUT (vermelho). Linha pontilhada representa o inicio do segundo dia.

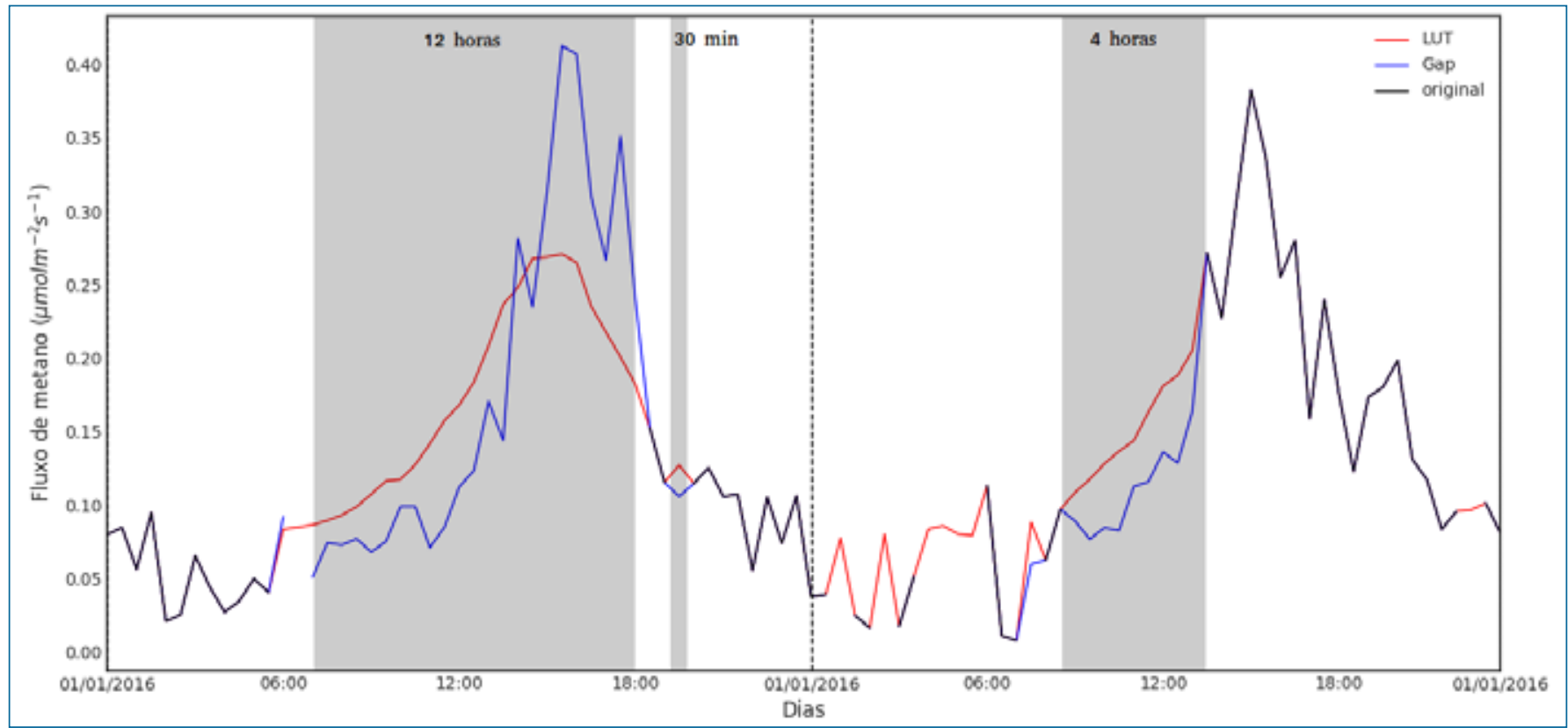

\section{Resultados}

O teste com diferentes técnicas de preenchimento do $\mathrm{fCH}_{4}$ ocorreu de modo que foram feitas falhas artificiais de 30 minutos, 4 horas e 12 horas.

Devido a grande diferença entre os valores diurno e noturno do $\mathrm{fCH}_{4}$ (dados não mostrados, embora este comportamento pode ser observado na figura 1), a análise realizada de forma distinta entre estes períodos. Na tabela 3, são apresentados os valores de $\mathrm{r}^{2}$ e RMSE para os diferentes tamanhos de lacunas geradas artificialmente referente aos valores diurnos e noturnos. Para o período diurno, o LI obteve qualificação "Bom"para preenchimento de falhas de meia hora. Já para falhas de 4 horas o LI e LUT obtiveram qualificação "Médio". Para falhas de 12 horas a técnica de MDV e LUT obtiveram qualificação "Médio". De forma geral, todas as técnicas usadas apresentaram uma menor capacidade dos modelos em representar os dados observados logo, nenhuma técnica obteve qualificação "Muito Bom".

Para o período noturno (tabela 3), em todos os casos, as técnicas obtém qualificação "ruim". Isto provavelmente pode estar ligado ao fato de que a baixa turbulência noturna compromete a qualidade dos dados de $\mathrm{fCH}_{4}$ obtidos a partir do método de EC (Acevedo et al. (2006)). Na comparação dos desempenhos das técnicas tanto para o período noturno quanto diurno, a técnica LUT se sobrepõe das demais, pois a técnica consegue representar melhor falhas de 12 horas, o que não ocorre com a LI e com baixa $\mathrm{r}^{2}$ para MDV noturno.

A figura 1 mostra os dados originais do $\left(\mathrm{fCH}_{4}\right)$, as falhas criadas artificialmente e os fluxos estimados pela técnica de LUT para dois dias consecutivos. No gap de 12 horas a técnica não conseguiu representar o pico do fluxo observado. No gap de meia hora a técnica se mostrou eficaz. No gap de 4 horas a técnica obteve valores maiores que o observado, causando assim, uma superestimativa do $\mathrm{fCH}_{4}$. 
Tabela 3: Comparação do $\mathrm{r}^{2}$ e RMSE entre as diferentes técnicas para falhas artificiais de meia hora, 4 horas e 12 horas no sítio de Cachoeira do Sul durante o período diurno e noturno.

\begin{tabular}{|c|c|c|c|c|c|c|c|}
\hline & \multicolumn{3}{|c|}{$\mathbf{r}^{2}$} & \multicolumn{3}{|c|}{ RMSE } \\
\hline & & $30 \min$ & $\overline{4 h}$ & $12 \mathrm{~h}$ & $30 \mathrm{~min}$ & $\overline{4 h}$ & $12 \mathrm{~h}$ \\
\hline \multirow{3}{*}{$\underline{\text { Diurno }}$} & LI & 0.82 & 0.69 & $\mathrm{n} / \mathrm{a}$ & 0.09 & 0.13 & $\mathrm{n} / \mathrm{a}$ \\
\hline & MDV & 0.45 & 0.49 & 0.60 & 0.17 & 0.16 & 0.13 \\
\hline & LUT & 0.54 & 0.51 & 0.67 & 0.16 & 0.13 & 0.13 \\
\hline & & \multicolumn{3}{|c|}{$\mathbf{r}^{2}$} & \multicolumn{3}{|c|}{ RMSE } \\
\hline & & $30 \min$ & $4 h$ & $12 \mathrm{~h}$ & $30 \mathrm{~min}$ & $\overline{4 h}$ & $12 h$ \\
\hline \multirow{3}{*}{ Noturno } & LI & 0.22 & 0.29 & $\mathrm{n} / \mathrm{a}$ & 0.10 & 0.06 & $\mathrm{n} / \mathrm{a}$ \\
\hline & MDV & 0.02 & 0.30 & 0.0009 & 0.13 & 0.06 & 0.08 \\
\hline & LUT & 0.19 & 0.18 & 0.18 & 0.11 & 0.09 & 0.05 \\
\hline
\end{tabular}

\section{Conclusão}

Neste trabalho, diferentes técnicas de preenchimento de fluxos de metano $\left(\mathrm{fCH}_{4}\right)$, estimados pelo método de EC, foram testadas As técnicas de preenchimentos foram aplicadas para gaps de 30 minutos, 4 horas e 12 horas, criadas aleatoriamente. A técnica de LI apenas preencheu gaps de 30 minutos e 4 horas devido sua baixa representatividade para gaps maiores que 4 horas.

Os preenchimentos realizados pela técnica LUT obtiveram os melhores resultados, na média, para todas as falhas, aproximando os dados de fluxo gerados aos dados originais, com melhores coeficiente de determinação para o período diurno.

\section{Referências}

Acevedo, O. C., Moraes, O. L. L., Degrazia, G. A., Medeiros, L. E. (2006). Intermittency and the exchange of scalars in the nocturnal surface layer. Boundary-Layer Meteorology, 119(1), 41-55.

Alberto, M. C. R., Wassmann, R., Buresh, R. J., Quilty, J. R., Correa, T. Q., Sandro, J. M., Centeno, C. A. R. (2014). Measuring methane flux from irrigated rice fields by eddy covariance method using open-path gas analyzer. Field Crops Research, 160, $12-21$.

Alvalá, P. C., Kirchhoff, V. W., Pavão, H. G. (1999). Metano Na Atmosfera. Biotecnologia Ciência \& Desenvolvimento, 2 , 39-43.

Aubinet, M., Vesala, T., Papale, D. (2012). Eddy Covariance Measurements Over Urban Areas. Em: Eddy Covariance A Practical Guide to Measurement and Data Analysis-, August, p 461.

Bhattacharyya, P., Neogi, S., Roy, K. S., Dash, P. K., Nayak, A. K., Mohapatra, T. (2014). Tropical low land rice ecosystem is a net carbon sink. Agriculture, Ecosystems and Environment, 189, 127-135.

BURBA G., ANDERSON D. (2005). A Brief practical Guide to Eddy Covariance Flux Measurements: Principles and workflow Examples for Scientific and industrial Application. LI-COR Biosciences.

CONAB: Companhia Nacional de Abastecimento (2016). Acompanhamento da Safra Brasileira de Grãos, vol 3. URL http: //www. conab.gov.br.

Dengel, S., Zona, D., Sachs, T., Aurela, M., Jammet, M., Parmentier, F. J. W., Oechel, W., Vesala, T. (2013). Testing the applicability of neural networks as a gap-filling method using $\mathrm{CH} 4$ flux data from high latitude wetlands. Biogeosciences, 10(12), 8185-8200.

Detto, M., Verfaillie, J., Anderson, F., Xu, L., Baldocchi, D. (2011). Comparing laser-based open- and closed-path gas analyzers to measure methane fluxes using the eddy covariance method. Agricultural and Forest Meteorology, 151(10), 1312-1324.

Diaz, M., Roberti, D. R. (2015). CÁLCULO DAS TROCAS DE CARBONO NUM AGROECOSSISTEMA DE ARROZ COM APLICAÇÃO DE TÉCNICAS DE PREENCHIMENTO DE FALHAS. Ciência e Natura, 37(0), URL http: / / cas cavel ufsm.br/revistas/ojs-2.2.2/index.php/cienciaenatura/article/view/16211.

Embrapa, E. B. D. P. A. (2006). Sistema brasileiro de classificação de solos. 
Falge, E., Baldocchi, D., Olson, R. (2001). Gap filling strategies for long term energy flux data sets. Agricultural and Forest Meteorology, (107), 71-77, URL http://www.sciencedirect.com/science/article/pii/s 0168192300002355.

Foken, T., Göockede, M., Mauder, M., Mahrt, L., Amiro, B., Munger, W. (2004). Post-Field Data Quality Control. Em: Handbook of Micrometeorology, pp. 181-208, URL http://dx.doi.org/10.1007/1-4020-2265-4_9\%5Cnhttp: //1ink.springer.com/10.1007/1-4020-2265-4_9.

IPCC (2013). Working Group I Contribution to the IPCC Fifth Assessment Report, Climate Change 2013: The Physical Science Basis. Ipcc, AR5(March 2013), 2014, URL http://ipccwg1.jp/AR4/meeting/pdf/SyR0407-siryo2 . pdf $\left\{\frac{\circ}{\circ}\right.$ 5Cnhttp://scholar.google.com/scholar?hl=en $\{\&\}$ btnG=Search $\{\&\} q=i n t i t l e:$ IPCC, +2014:+Climate+Change+2014:+Impacts, +Adaptation, +and+Vulnerability .+Part+A: +Global+and+Sectoral+Aspects. +Contribution+of+Working+Gro, arXiv:1011.1669v3.

Moffat, A. M., Papale, D., Reichstein, M., Hollinger, D. Y., Richardson, A. D., Barr, A. G., Beckstein, C., Braswell, B. H., Churkina, G., Desai, A. R., Falge, E., Gove, J. H., Heimann, M., Hui, D., Jarvis, A. J., Kattge, J., Noormets, A., Stauch, V. J. (2007). Comprehensive comparison of gap-filling techniques for eddy covariance net carbon fluxes. Agricultural and Forest Meteorology, 147(3-4), 209-232, URL http: / / linkinghub. elsevier.com/retrieve/pii/S016819230700216X.

Taiz, L., Zeiger, E. (2006). Fisiología Vegetal. Em: Fisiología Vegetal, pp. 536,537.

Lucas Augusto Fagundes

Universidade Federal de Santa Maria E-mail: lucasaugustofagundes@gmail.com

Cristiano Maboni

Universidade Federal de Santa Maria E-mail: cristianomaboni@hotmail.com

Maria Eduarda Pinheiro

Universidade Federal de Santa Maria E-mail: mariaeduardafisica@gmail.com

Josue Sehnem

Universidade Federal de Santa Maria E-mail: josue@sehnem.com

Marcelo Diaz

Universidade Federal de Santa Maria E-mail: marbdiaz@gmail.com

Debora Regina Roberti

Universidade Federal de Santa Maria E-mail: debora@ufsm.br 